\title{
Effect of Multi-Electrode Configurations on Accuracy of Rotor Detection in the Atria
}

\author{
Laura Martínez ${ }^{1}$, Lucía Romero ${ }^{1}$, Ana Ferrer ${ }^{1}$, José Jalife² ${ }^{2}$ Omer Berenfeld ${ }^{2}$, Javier Saiz ${ }^{1}$ \\ ${ }^{1}$ Ci2B, Universitat Politècnica de València, Valencia, Spain \\ ${ }^{2}$ Center for Arrhythmia Research, University of Michigan, Ann Arbor, Michigan, USA
}

\begin{abstract}
Multi-electrode catheters are increasingly being used to localize rotors that drive atrial fibrillation. Our objective is to analyze the effects of the multi-electrodes geometrical configuration on the accuracy of localizing rotors using computer simulations.

We simulated rotor detection in: 1) a $3 D$ cube of tissue and various multi-electrode array configurations, varying the electrodes-to-tissue distance ( $d=0.9$ to $19.8 \mathrm{~mm}$ ) and the inter-electrode distance ( $d_{i e}=0.9$ to $18 \mathrm{~mm}$ ), and 2) a $3 D$ realistic atrial model and an intra-cardiac basket catheter in two locations inside the right atrium (RA). Rotors were detected by localizing phase singularity points (PSs) on phase maps based on the Hilbert transform.

Our simulations showed that for the 3D block, the best PS trajectory detection (sensitivity $~ 85 \%$ ) was obtained at $d=d_{i e}=0.9 \mathrm{~mm}$. Increasing $d$ decreased the sensitivity. However, for the highest $d_{i e}$ it increased from $30 \%$ to $53 \%$ by increasing $d$. For the rotor simulated in the $R A$, the basket achieved $90 \%$ accuracy in detection of the rotor in a position where the averaged distance between the electrodes and the rotor was $0.96 \mathrm{~cm}$. The accuracy decreased to $35 \%$ when the distance increased to $3.05 \mathrm{~cm}$. Moreover, false rotors appeared on the basket's phase maps due to the far field sources and the EGMs interpolation.
\end{abstract}

\section{Introduction}

Atrial fibrillation (AF), one of the most common cardiac arrhythmias in the clinical practice [1], is suggested to be driven often by rotors which have become ablation target for its terminate [2]. These new patient-specific ablation procedures depend on the atrial activation of each patient and have improved the rate of success compared to the traditional and patient-independent pulmonary veins isolation [1]. However, this improved success rate is still sub-optimal and it is desirable to develop new technologies to help physicians identify and locate the rotor drivers with the aim of improving the outcomes in the ablation procedures.

Nowadays, multi-electrode systems are increasingly being used to map the atrial electrical activity in humans and localize those rotors. The minimum inter-electrode distance $\left(\mathrm{d}_{\mathrm{ie}}\right)$ necessary to detect the rotors has been studied already [3], but the common variation in distance between the electrodes and the tissue (d) present in the clinical setting wasn't considered.

In the present work we use simulations on 3D computer models to characterize the effects of the multi-electrodes geometrical configuration, including variations in both the $\mathrm{d}_{\mathrm{ie}}$ and $\mathrm{d}$ distances, on the accuracy of localizing rotors.

\section{Methods}

\subsection{Computer simulations}

In order to analyze the effects of the geometrical configuration in the multi-electrodes systems, we performed computer simulations in a 3D cube of tissue and a $3 \mathrm{D}$ realistic atrial model. In the first case, a simulation in paroxysmal AF (pAF) conditions was carried out to reproduce functional reentries in the left atrium (LA). The CRN model [4] was modified by the $\mathrm{I}_{\mathrm{KACh}}$ ionic current of the Grandi et al. model [5] and used to reproduce the cellular electrical activity under pAF conditions. The maximum conductance of the $\mathrm{I}_{\mathrm{Kr}}$ and $\mathrm{I}_{\mathrm{K} 1}$ ionic channels was modified with respect to the original model to adjust the atrial heterogeneity and the electrical remodeling under pAF conditions in the LA, respectively (see [6] for further information). In case of the 3D atrial model, a simulation in chronic AF conditions (cAF) was carried out. The maximum conductance of several ionic currents was adjusted in the CRN model to reproduce the atrial heterogeneity (similarly to [7]) and the electrical remodeling under cAF conditions [8-12], as shown in Table 1. The right atrium (RA) remodeling was applied to all the structures within the RA, while the LA remodeling was applied to the structures within the LA.

In the simulation with a 3D block of tissue, we analyzed rotors detection through various multi-electrode array configurations, varying the electrodes-to-tissue distance (d) from $0.9 \mathrm{~mm}$ to $19.8 \mathrm{~mm}$ and the inter-electrode distance $\left(\mathrm{d}_{\mathrm{ie}}\right)$ from $0.9 \mathrm{~mm}$ to $18 \mathrm{~mm}$. An example of these configurations is shown in Figure 1A. In the simulation 
with the 3D atrial model, rotor detection was analyzed through an intra-cardiac basket-type mapping catheter of 64 electrodes $(8 \times 8)$ positioned at two locations inside the RA (Figure 1B).

Table 1. Changes in the ionic currents of the CRN model to reproduce: atrial heterogeneity (left) and remodeling under cAF conditions (right).

\begin{tabular}{|c|c|c|c|c|c|c|}
\hline Heterog*. & Ito & ICaL & $\mathbf{I}_{\mathrm{Kr}}$ & cAF & RA & LA \\
\hline RA/PM & 1.00 & 1.00 & 1.00 & g to & $-45 \%$ & $-75 \%$ \\
\hline CT/BBRA & 1.00 & 1.67 & 1.00 & gCaL & $-65 \%$ & $-65 \%$ \\
\hline TVR & 1.00 & 0.67 & 1.53 & $\mathbf{g}_{\mathrm{K} 1}$ & $+100 \%$ & $+100 \%$ \\
\hline RAA & 0.68 & 1.00 & 1.00 & gKur & $-60 \%$ & $-45 \%$ \\
\hline LA & 1.00 & 1.00 & 1.60 & gKs & $+150 \%$ & $+100 \%$ \\
\hline BBLA & 1.00 & 1.67 & 1.60 & & & \\
\hline MVR & 1.00 & 0.67 & 2.44 & & & \\
\hline LAA & 0.68 & 1.00 & 1.60 & & & \\
\hline PV & 1.00 & 1.00 & 2.20 & & & \\
\hline
\end{tabular}
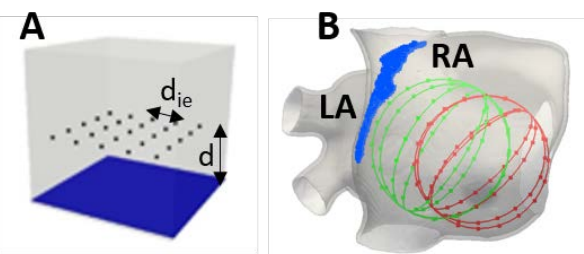

Figure 1. A) Multi-electrode array: configuration for $\mathrm{d}=19.8 \mathrm{~mm}$ and $\mathrm{d}_{\mathrm{ie}}=9 \mathrm{~mm}$. Electrodes, black; atrial endocardium, blue. B) Intra-cardiac spherical basket catheter inside the RA: position 1 (green) and position 2 (red). Simulated rotor meandering area on the endocardium of the RA is in blue.

In both simulations, the electrical propagation of action potentials (AP) was solved by the mono-domain formulation and the unipolar electrograms (EGMs) were computed through the extracellular electrical potentials $\left(\varphi_{\mathrm{e}}\right)$ calculation by an approximation of the bidomain formulation [13]. The EGMs were computed in the atrial blood cavity at the specific coordinates where the electrodes of the multi-electrode array and the basket catheter were located. The EGMs were linearly interpolated to obtain a continue visualization of the phase maps, based on the Hilbert transform, and a more accurate tracking of the trajectories through the phase singularities (PS) detection. For further information about the methodology see [6].

\section{Results and discussion}

To establish a clear baseline understanding we first evaluated the effects of the geometrical configuration of the multi-electrodes systems on the accuracy of localizing rotors in a 3D cube. We simulated a couple of rotors in a $3 \mathrm{D}$ cube of atrial tissue and analyzed their detection by multi-electrodes arrays with varying configurations. Following we studied a more complex and realistic mapping where we simulated a rotor in our 3D atrial model and its detection was evaluated by an intra-cardiac 64electrodes spherical basket-type mapping catheter positioned in two different locations inside the RA.

For the simulations on the 3D block model, Figure 2 is presenting snapshots of the voltage and phase maps obtained at the endocardium at $\mathrm{t}=600 \mathrm{~ms}$, as well as the PS detections describing the rotors' trajectories for the duration of the simulation.

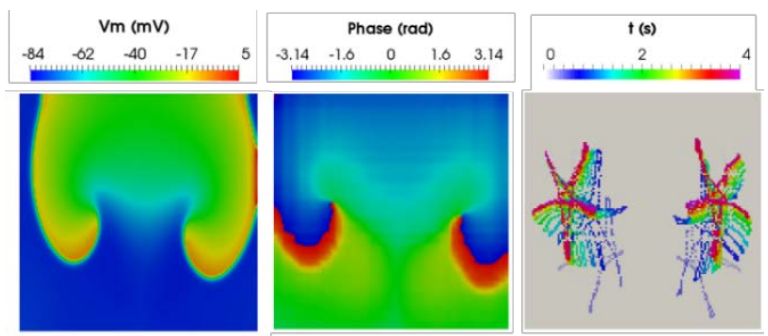

Figure 2. Color-coded voltage (left) and phase map (center) snapshots at $\mathrm{t}=600 \mathrm{~ms}$, and rotor PS's trajectory detection (right) for the whole simulation duration (4 sec), in the 3D block model of the atrial tissue and cavity.

Phase maps obtained by each multi-electrodes array configuration following computation of the EGMs and interpolations are shown in Figure 3. As expected, the phase maps of the multi-electrode array configuration with the minimal $\mathrm{d}=\mathrm{d}_{\mathrm{ie}}=0.9 \mathrm{~mm}$ is the one that resembles most the endocardial phase map shown in Figure 2. However, the effects of increasing $d_{\text {ie }}$ on the phase maps seem to be stronger when the electrode is closer to the tissue (smaller d).

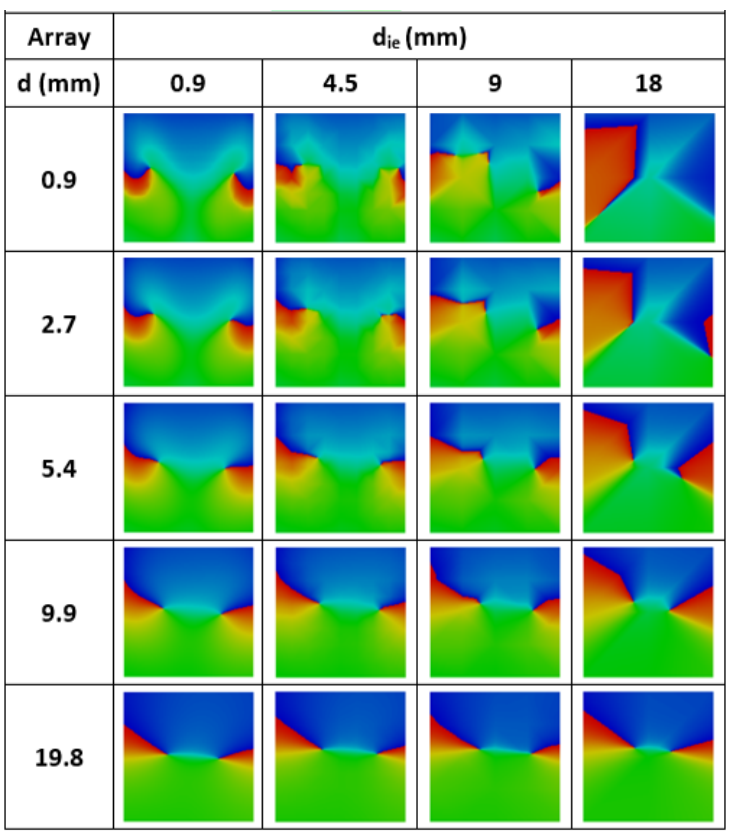

Figure 3. Phase maps corresponding to each multielectrodes array configuration.

Thereafter, rotors were tracked through the PSs detections for each multi-electrodes array configuration. 
The results are summarized in Figure 4. Sensitivity and specificity were calculated by matching the trajectories detected by the arrays and the ones detected on the endocardium. As expected from Figure 3, Figure 4 reveals that the best trajectory detection was obtained at $\mathrm{d}=\mathrm{d}_{\mathrm{ie}}=0.9 \mathrm{~mm}$, with a sensitivity $\sim 85 \%$. Moreover, increasing $d$ decreased the sensitivity (to a minimum of $58 \%$ at $\mathrm{d}=19.8 \mathrm{~mm}$ ). However, surprisingly for the highest $\mathrm{d}_{\mathrm{ie}}(18 \mathrm{~mm})$ the sensitivity increased from $30 \%$ to $53 \%$ by increasing d. Also, specificity decreased by increasing $d$ and $\mathrm{d}_{\mathrm{ie}}$. Nevertheless it was $>84 \%$ in all cases.

\begin{tabular}{|c|c|c|c|c|}
\hline Array & \multicolumn{4}{|c|}{$\mathrm{d}_{\mathrm{ie}}(\mathrm{mm})$} \\
\hline $\mathrm{d}(\mathrm{mm})$ & 0.9 & 4.5 & 9 & 18 \\
\hline 0.9 & $\begin{array}{l}y \\
\text { Sens }=84.7 \% \\
\text { Spe }=97.7 \%\end{array}$ & $\begin{array}{l}\text { of } \frac{5}{3 \%} \\
\text { Sens }=66.3 \% \\
\text { Spe }=95.0 \%\end{array}$ & $\begin{array}{l}\text { Sens }=42.3 \% \\
\text { Spe }=87.4 \%\end{array}$ & $\begin{array}{c}\text { Sens }=29.9 \% \\
\text { Spe }=84.3 \%\end{array}$ \\
\hline 2.7 & $\begin{array}{l}\text { Xy } \\
\text { Sens }=68.5 \% \\
\text { Spe }=95.0 \%\end{array}$ & $\begin{array}{l}\text { the } \\
\text { Sens }=62.9 \% \\
\text { Spe }=94.3 \%\end{array}$ & $\begin{array}{l}\text { Sens }=49.7 \% \\
\text { Spe }=90.4 \%\end{array}$ & $\begin{array}{l}\text { Sens }=40.0 \% \\
\text { Spe }=88.6 \%\end{array}$ \\
\hline 5.4 & $\begin{array}{c}\text { Sens }=57.4 \% \\
\text { Spe }=93.1 \%\end{array}$ & $\begin{array}{l}\text { Sens }=56.5 \% \\
\text { Spe }=93.0 \%\end{array}$ & $\begin{array}{l}\text { Sens }=50.8 \% \\
\text { Spe }=91.0 \%\end{array}$ & $\begin{array}{l}\text { Sens }=44.3 \% \\
\text { Spe }=91.3 \%\end{array}$ \\
\hline 9.9 & $\begin{array}{l}\text { Sens }=57.1 \% \\
\text { Spe }=93.4 \%\end{array}$ & $\begin{array}{l}\text { Sens }=58.1 \% \\
\text { Spe }=93.7 \%\end{array}$ & $\begin{array}{l}\text { Sens }=52.0 \% \\
\text { Spe }=92.0 \%\end{array}$ & $\begin{array}{l}\text { Sens }=49.4 \% \\
\text { Spe }=94.1 \%\end{array}$ \\
\hline 19.8 & $\begin{array}{l}\text { Sens }=58.8 \% \\
\text { Spe }=95.8 \%\end{array}$ & $\begin{array}{l}\text { Sens }=59.5 \% \\
\text { Spe }=95.8 \%\end{array}$ & $\begin{array}{c}\text { Sens }=53.5 \% \\
\text { Spe }=93.8 \%\end{array}$ & $\begin{array}{l}\text { Sens }=53.0 \% \\
\text { Spe }=95.4 \%\end{array}$ \\
\hline
\end{tabular}

Figure 4. Rotors' trajectories detected by each multielectrodes array configuration.

Finally, to evaluate rotor detection under more realistic conditions, we used a 3D atrial model and an intra-cardiac basket-type mapping catheter. In this case, our simulation yielded a stable rotor along the crista terminalis. Figure 5A illustrates the AP propagation (top) and phase map (bottom) in the 3D atrial model at $t=5000 \mathrm{~ms}$. This figure highlights that the phase map is intimately related to the AP propagation in the endocardium. Then, the EGMs were computed on the coordinates where the electrodes of the basket were placed and also were linearly interpolated for phase maps visualization and the rotor's tip detection. Figure $5 \mathrm{~B}$ shows the open rendering phase maps of basket in the two considered positions (Figure 1). The averaged distance between the electrodes and the rotor meandering area was $0.96 \mathrm{~cm}$ and $3.05 \mathrm{~cm}$ for positions 1 and 2, respectively. The trajectories of the PSs are superimposed on the basket maps in white dots. When comparing the phase maps and the PSs' trajectories registered by the basket to the phase maps in the endocardium, it turns out that the rotor is detected during the $90 \%$ and the $35 \%$ of the simulation time span in the position 1 and 2, respectively. Importantly, false rotors appeared on the basket's phase maps as a consequence of the influence of the far field sources when electrodes were not in fullcontact with the tissue, as well as the EGMs interpolation.

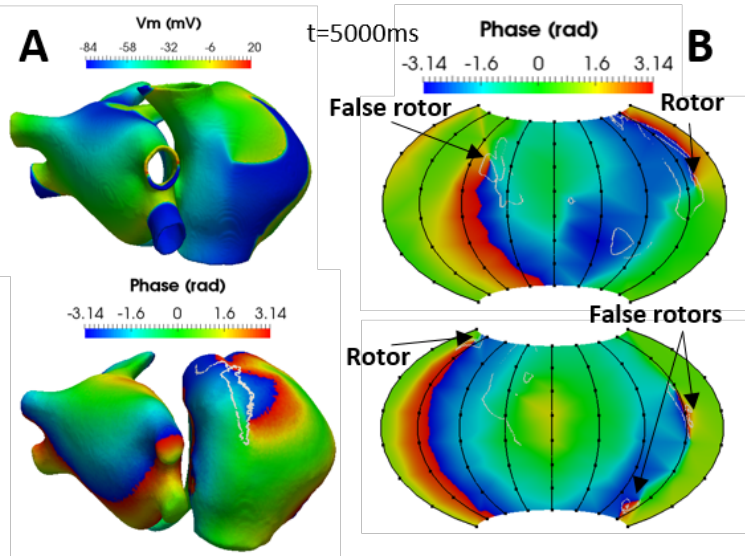

Figure 5. Snapshots at $\mathrm{t}=5000 \mathrm{~ms}$ of the: A) AP propagation (top) and phase maps (bottom) in the 3D atrial model; B) phase maps built from the basket EGMs in position 1 (top) and 2 (bottom) described in Figure 1. PS: white dots.

On the one hand, our results obtained with the 3D block of atrial tissue and cavity suggests that when the spatial resolution of the electrodes was poor, rotors' detection improved by increasing the distance $d$ to the tissue; in this study it occurred for $d_{i e} \geq 9 \mathrm{~mm}$ where the sensitivity and the specificity started to increase when increasing $d$. Nevertheless, $\mathrm{d}_{\mathrm{ie}}<9 \mathrm{~mm}$ seemed to be a sufficient spatial resolution to detect rotors with a relatively high sensitivity of $\sim 85 \%$ for low distances to the tissue ( $\mathrm{d}=0.9 \mathrm{~mm}$ ). These results are in accordance to a previous study in which it was demonstrated that rotors could be detected with a spatial resolution of $1 \mathrm{~mm}$ to $1 \mathrm{~cm}$ [3]. However here we provide further insight that the minimum spatial resolution depends on the distance between the array of electrodes and the tissue.

On the other hand, the results obtained in our simulations with a realistic 3D atrial model and a basket catheter, in which $\mathrm{d}_{\mathrm{ie}}$ ranged from a few $\mathrm{mm}$ to $\sim 1 \mathrm{~cm}$, reveal that rotor detection was more accurate in the position 1 than in the position 2 (that is, closer to the rotor area), where the rotor was detected during a higher portion of the simulation time span. The best detection of the rotor (90\%) occurred when the averaged distance between the electrodes to the rotor meandering area was $\sim 1 \mathrm{~cm}$, while the worst detection occurred when it was $\sim 3 \mathrm{~cm}$ away. 
Overall, the results obtained with the simple 3D block of atrial tissue and cavity are not directly applicable to more complex atrial geometries and multi-electrodes configurations, as for example in the spherical basket catheter case. In the case of the basket $\mathrm{d}_{\mathrm{ie}}$ is variable and the distance between the electrodes and the endocardium, and also to the rotor are non-constant. However, most of the electrodes have a $d_{i e}$ in the range of a few mm (only the electrodes at the equator are separated by $\sim 1 \mathrm{~cm}$ ) and this spatial resolution is above the threshold in our 3D block analysis $\left(\mathrm{d}_{\mathrm{ie}}<9 \mathrm{~mm}\right)$. Accordingly, it is reasonable that the more accurate detection of rotors in the atria with a basket happens for position 1, in which the catheter and electrodes are closer to the rotor.

\section{Conclusion}

Localization of a rotor with high density multi-electrode arrays placed in a cavity parallel to a flat endocardium can be accurate when the array is placed closest to the endocardium. For low density arrays, however, accuracy can be maintained by increasing the distance to the tissue. Additionally, atrial rotor detection by spherical basket catheter is most accurate when the average distance between the electrodes and the rotor's trajectory is less than $1 \mathrm{~cm}$. Importantly, false rotors might appear on the basket's phase maps as a consequence of the far field sources and the interpolation of the EGMs.

\section{Acknowledgements}

This study was supported in part by grants from the "Programa Prometeo", Generalitat Valenciana, Award Number: 2016/088, from NIH (R01-HL118304) and from Medtronic.

\section{References}

[1] Calkins H, Kuck KH, Cappato R, Brugada J, Camm AJ, Edgerton J, et al. 2012 HRS/EHRA/ECAS expert consensus statement on catheter and surgical ablation of atrial fibrillation: recommendations for patient selection, procedural techniques, patient management and follow-up, definitions, endpoints, and research trial design. J Interv Card Electrophysiol. 2012;33: 171-257.

[2] Narayan SM, Shivkumar K, Krummen DE, Miller JM, Rappel W-J. Panoramic Electrophysiological Mapping but not Electrogram Morphology Identifies Stable Sources for Human Atrial Fibrillation. Stable Atrial Fibrillation Rotors and Focal Sources Relate Poorly to Fractionated Electrograms. Circ Arrhythm Electrophysiol. 2013;6: 58-67.

[3] Rappel WJ, Narayan SM. Theoretical considerations for mapping activation in human cardiac fibrillation. Chaos. 2013;23: 023113. doi:10.1063/1.4807098

[4] Courtemanche M, Ramirez RJ, Nattel S. Ionic mechanisms underlying human atrial action potential properties: insights from a mathematical model. Am J Physiol Heart Circ Physiol. 1998;275: 301-321.

[5] Grandi E, Pandit SV, Voigt N, Workman AJ, Dobrev $\mathrm{D}$, Jalife J, et al. Human atrial action potential and Ca2+ model: sinus rhythm and chronic atrial fibrillation. Circ Res. 2011; 109 (9): 1055-66.

[6] Martinez L, Jalife J, Berenfeld O, Saiz J. Are Multielectrode Arrays able to Differentiate Anatomical from Functional Reentries in an Excitable Sheet? Computing in Cardiology. 2015. pp. 865-868.

[7] Ferrer A, Sebastián R, Sánchez-Quintana D, Rodríguez JF, Godoy EJ, Martínez L, et al. Detailed Anatomical and Electrophysiological Models of Human Atria and Torso for the Simulation of Atrial Activation. Plos One. 2015;10: e0141573.

[8] Caballero R, de la Fuente MG, Gómez R, Barana A, Amorós I, Dolz-Gaitón P, et al. In Humans, Chronic Atrial Fibrillation Decreases the Transient Outward Current and Ultrarapid Component of the Delayed Rectifier Current Differentially on Each Atria and Increases the Slow Component of the Delayed Rectifier Current in Both. J Am Coll Cardiol. 2010;55: 2346-2354.

[9] Bosch RF, Zeng X, Grammer JB, Popovic K, Mewis C, Kühlkamp V. Ionic mechanisms of electrical remodeling in human atrial fibrillation. Cardiovascular Research. 1999;44: 121-131.

[10] Van Wagoner DR, Pond a L, Lamorgese M, Rossie SS, McCarthy PM, Nerbonne JM. Atrial L-type Ca2+ currents and human atrial fibrillation. Circ Res. 1999;85: 428-436.

[11] Workman AJ, Kane AK, Rankin AC. The contribution of ionic currents to changes in refractoriness of human atrial myocytes associated with chronic atrial fibrillation. Cardiovascular Research. 2001;52: 226-235.

[12] Dobrev D, Graf E, Wettwer E, Himmel HM, Hála O, Doerfel C, et al. Molecular basis of downregulation of G-protein-coupled inward rectifying $\mathrm{K}(+)$ current ( $\mathrm{I}(\mathrm{K}, \mathrm{ACh})$ in chronic human atrial fibrillation: decrease in GIRK4 mRNA correlates with reduced $\mathrm{I}(\mathrm{K}, \mathrm{ACh})$ and muscarinic receptor-mediated shortening of action potentials. Circulation. 2001;104: 2551-2557.

[13] Keller DUJ, Weber FM, Seemann G, Dössel O. Ranking the influence of tissue conductivities on forward-calculated ecgs. IEEE Transactions on Biomedical Engineering. 2010;57: 1568-1576.

Address for correspondence:

Laura Martínez Mateu

Ci2B - Ciudad Politécnica de la Innovación, Cubo Azul Edif. 8B, Acceso N. Camino de Vera s/n, 46022 - Valencia (Spain)

laumarma@ci2b.upv.es 